\title{
Establishing a Combined Neonatal and Paediatric Transport System From Scratch
}

Stephen Hancock, MBChB, DCH, MRCP, FRCPCH ${ }^{1,2, *}$ Catherine Harrison, BMedSCi, BM, BS, MRCP, DTM\&H, FRCPCH ${ }^{1}$

\author{
Address \\ ${ }^{*}, 1$ Embrace Yorkshire \& Humber Infant \& Children's Transport Service, Unit 7 \\ Capitol Close, Capitol Business Park, Barnsley, S75 3UB, UK \\ Email: stephen.hancock@sch.nhs.uk \\ ${ }^{2}$ Honorary Senior Lecturer, University of Sheffield School of Medicine, Sheffield, \\ UK
}

Published online: 2 February 2018

(C) The Author(s) 2018. This article is an open access publication

This article is part of the Topical Collection on Transport Medicine

Keywords Transport $\cdot$ Neonatal $\cdot$ Paediatric $\cdot$ Interfacility $\cdot$ Network

\section{Abstract}

Purpose of review Centralisation of neonatal and paediatric intensive care over the last 30 years has led to a significant increase in the numbers of newborn babies and children requiring interfacility transport. Many early ad hoc transport teams have evolved into specialised services providing mobile ICUs capable of providing state of the art critical care. This paper focuses on the development of combined neonatal and paediatric transport teams which has been driven by a pragmatic analysis of the benefits flowing from critical mass, economics and clinical cross fertilisation.

Recent findings There is a growing body of evidence that specialist transport teams have better outcomes in terms of morbidity and mortality; however, anecdotal evidence would suggest that the benefits of a combined team are somewhat counterbalanced by the significant challenges of the wide scope of care and logistical complexity.

Summary Establishing a combined service requires vision, time, hard work and support from the clinical and health care commissioning communities. Success of the project will depend on strong clinical leadership, a dedicated highly skilled group of professionals who share the vision, financial support and a foundation based on quality and safety. Given the high risk nature of the activity, any new service will need to benchmark against recognised standards and key performance metrics to ensure the best care possible is provided to this most vulnerable group of patients. 


\section{Introduction}

Critical care transport has become increasingly important to the outcomes of patients in healthcare systems around the world as care becomes centralised in specialist centres. In the USA alone, more than 200,000 newborns, infants and children are transported from local hospitals to regional hospitals for inpatient specialty care [1].

Morbidity and adverse events during transport have been shown to be reduced by specialised teams, from $20 \%$ in non-specialised teams to $2 \%$ [2]. Seventy-five percent children in an early study by Barry et al. had an adverse clinical event during transfer leading to a higher mortality rate after admission to PICU [3]. More recent work using UK data showed the use of specialised teams improves survival (OR 0.58, CI 0.39-0.87) [4].

Orr's seminal paper in 2009 added weight to earlier work that specialised teams must be used for transport [5]. In this large study, mortality rates in non-specialised teams were $23 \%$ compared to $9 \%$ after adjusting for illness severity. The use of a non-specialised team had an independent association with adverse events, most commonly airway, and mortality was also increased.

McPherson's review article reminded us that speed is not everything in paediatric medical transport and that every seven times a generalised team is used rather than a specialised team, a child may die [6].
The data on speed of transfer are variable and Voss et al. showed that patients moved with untrained referring hospital staff took longer had higher incidence of complications during transfer, and more interventions were needed on arrival at paediatric ICU [7]. More recent data showed that generalised teams were quicker, but patients showed a worsening in both Pediatric Trauma Scores as well as Glasgow Coma Scales scores from referral to arrival, despite a similar severity of injury [8$\bullet$.

This has led to transports being carried out by specialised teams to meet the needs of their local populations and healthcare infrastructure, and inevitably, this has been heavily influenced by the economics of healthcare. Initially, these specialised teams were individualised neonatal or paediatric teams with no clinical cross over but over time, teams have had to reflect on, design and develop services in line with local and regional need. To many it seems natural to consider combining neonatal and paediatric transport systems to benefit from the critical mass, shared infrastructure and clinical cross fertilisation. To others, the challenges of the wide scope of care, ranging from extreme premature neonates to adult-sized teenagers, seem too great.

In this paper, we describe the process of combining services from scratch, focussing on our experiences in the Yorkshire \& Humber $(\mathrm{Y} \& \mathrm{H})$ region of the UK and reflecting on the experiences of others across the world.

\section{The Case for Change}

Generally, investment in, and development of, intensive care facilities for neonates and children came before the spotlight fell on transport infrastructure. Rapid growth in neonatal intensive care in the 1970s and paediatric intensive care in the 1990s led to a need for patients to be transferred to receive the right care in the right place at the right time. Initially, responsibility for transport fell to the referring team but it became clear that this was not in anyone's interest, putting undue pressure on stretched resources in smaller hospitals and creating multiple low frequency, high-risk events. In response to this anecdotal evidence, centralised neonatal transport systems began to develop in the 1980s, mainly out of the tertiary centres. However, these were often still ad hoc in nature, hence the popular term in the UK of 'flying squad' [9]. Similar initiatives followed in paediatric intensive care and the concept of the 'retrieval' team was born [10]. As these teams became more established, with a core group of trained and experienced staff using specific equipment and, in some cases, dedicated vehicles, there was evidence developing that outcomes were in-fact better for 
patients transferred by these specialist teams [10]. This evidence prompted the injection of healthcare commissioning funding to provide specialist teams that were numerically and/or physically separate allowing them to become 'go at all times' rather than 'go if able' services dependent on bed availability.

The situation in $\mathrm{Y} \& \mathrm{H}$ mirrored this description of transport team development and had kept pace with the changes in transport in other regions of the UK. However, it became stalled at the stage of specialist teams, which were 'go if able' and bed dependent in the late 1990s. During this time, a number of individuals gained experience in transport systems in Australia, where the challenges of geography and distance had created a drive to fully funded, regional transport teams with access to state of the art equipment and vehicles. In particular, the focus fell on New South Wales where the NETS team (Neonatal \& paediatric Emergency Transport Service) had combined the neonatal and paediatric retrieval services in Sydney in 1995. In 1999, the NETS team reported their early experience in an important paper challenging clinicians and healthcare planners in the UK to consider similar system change [11]. To achieve the desired critical mass of activity, the authors argued that amalgamating neonatal and paediatric teams was desirable and that an autonomous body dealing with the transport process could bring advantages in fast response times, improved team experience and probably lower morbidity rates. As a result of the experience gained in Australia and discussion stimulated by this paper, a small group in Leeds wrote a discussion document in 1999 making the case for the development of a joint neonatal and paediatric transport team for Yorkshire. This document was submitted to healthcare commissioners-the journey had begun.

\section{Building a Consensus}

Despite the involvement of two senior clinicians from both NIC and PIC, that first document calling for change failed to gain traction. Opposition was based around a number of issues. In some ways, the proposal just seemed too much of a sea change, as Rashid et al. had indicated there was likely to be intense bureaucracy involved in the design of a transport team serving a number of localities [11]. There was also the issue of cost. At face value, stand-alone transport systems seem more expensive than integrated somewhat ad hoc models, where many of the costs were hidden, and in any case, it was seen that they were doing a reasonable job by many. Finally, there was a significant majority of clinical staff who thought that a combined service was neither possible, nor even desirable because of the clinical risks associated with the wide scope of care. It is probably this clinical factor that is the most frequently cited in arguments opposing moves to combined services, and it is to this issue that advocates of change need to look when attempting to build a consensus.

\section{The Importance of Data}

When setting up a service, it is vital to establish activity levels to allow for service modelling. During our planning period, data were obtained from the four existing transport teams to predict the activity of the new service. It had been clear for some time that there was a significant unmet need, but the exact size of 
Know Your Region

the problem wasn't known. Neighbouring transport teams were asked to provide data on transfer requests made to them from $Y \& H$. The local ambulance services submitted information on the numbers of children transferred with a medical crew, indicating possible numbers of high dependency level transfers. The intensive care units and local neonatal units provided data on the number of repatriations and transfers they carried out. Analysis of these submissions revealed that the four existing teams carried out 1500 transfers per year, and there was an additional unmet need of 500. Planning the new service was therefore based on an estimated 2000 annual transfers.

Once a service becomes operational, monitoring of activity allows evaluation of the effectiveness and efficiency of a service. As with all transport teams, there are seasonal variations in activity, especially in paediatric transfers. From analysing our data in detail with respect to the frequency and timing of referrals, we have been able to model our service in terms of team provision by flexing up in the winter from five teams per $24 \mathrm{~h}$ period to six. Robust data collection also allows for benchmarking against other services. There are national and international organisations that provide this platform for data submission, analysis and comparison.

PICANET [12] and UK NTG [13] have allowed our service to submit data and compare activity and severity of illness in our patient groups as well as service infrastructure. Data submission to the Ground and Air Medical qUality Transport (GAMUT) database has allowed tracking, reporting and analysis of our service's performance on transport-specific quality metrics by comparing it to other teams worldwide [14]. The beauty of this for developing combined services which, like Embrace, may be working in relative isolation is that they can benchmark against services with a similar scope. This has allowed our service to focus on specific Quality Improvement work streams. Comparison data have also been pivotal in service development in the procurement of new equipment to improve performance. Aiming to be as transparent as possible, these data are all publically available online [15].

As well as activity data, regional statistics are important to allow full understanding and appreciation of patient groups.

$\mathrm{Y} \& \mathrm{H}$ has a population of 5.2 million people covering an area of $15,420 \mathrm{~km}$ [16]. The region as a whole is one of the more deprived areas in England with an infant mortality rate of 4.3 per 1000 compared to the UK average of 3.9. The perinatal mortality rate is $7 / 1000$ [17]. Ethnic diversity leads to some specific healthcare challenges, such as relatively high rates of rare metabolic disorders. There is a contrast between the three main metropolitan areas and a significant remote and rural community.

Local geographical information including locations of hospitals, road networks and densities of population hubs play a vital part in planning where services should be located. Our transport base is equidistant between the two regional paediatric intensive care units and adjacent to the major road network allowing quick access to the hospitals we serve. Access to air transport options is an important mechanism to deliver equitable healthcare and should be a consideration when planning services. 


\section{Option Appraisal—Model of Service}

When designing a new service, different models need to be considered to decide which has the best fit for the required needs.

An option appraisal involving clinicians, managers and healthcare commissioners needs to be carried out. Levels of integration between neonates and paediatrics, shared infrastructure and numbers and locations of transport bases all need to be considered. Weighted scoring of the various options presented and involvement of all stakeholders will help ensure the most equitable decision making. In $\mathrm{Y} \& \mathrm{H}$, the decision was to develop a combined regional neonatal and paediatric transport service. In addition, there was agreement that the service would be based off-site, separate from any hospital facility.

To define what was needed for our combined service, two strategic documents were used: Toolkit for High Quality Neonatal Services [18] and Paediatric Intensive Care Society (PICS) Standards for the Care of Critically Ill Children (updated 2015) [19]. These documents allowed us to plan not only logistical and operational aspects of the service, but also helped us focus on family needs and equity of access to specialised services. A non-clinical project manager was employed to oversee the development of the service and working groups were set up. As detailed above, the concept of a combined neonatal and paediatric transport service was novel in the UK at this time, and with that came a degree of anxiety and uncertainty about success. Therefore, participation of all current providers was essential. Clinicians were invited from local district general hospitals and intensive care units to be part of the planning and development process, alongside managers, healthcare commissioners, and local ambulance providers. Public engagement also occurred during the planning and development phase.

Work stream groups were established to create operational guidelines, clinical guidelines, equipment and ambulance specifications, financial plans and staffing requirements.

\section{Show me the Money}

Throughout the planning stage it is crucial to consider what financial support and input will be needed.

The operational specification in the Toolkit [18] was invaluable in drawing up equipment lists to allow detailed financial calculations for set up costs.

Predicted activity data allowed workforce planning to establish the number of staff needed to provide a $24 \mathrm{~h}$ service. A tendering process was used to establish a host organisation. Service level agreements were put in place with ambulance providers.

Different settings will have different financial approaches and models but the need for detailed plans and contracts regarding replacement equipment and vehicles cannot be underestimated. Service level agreements will be used for some independent contractors. Close liaison with finance and contract departments during the development of the service is critical. 


\section{Build and They Shall Come}

The transition from separate services to a combined model has to be carefully planned. Making a firm decision to combine and then working to an implementation deadline has clear advantages but is not without risk. In $Y \& H$, the new team initially provided paediatric transport services, while supporting the existing neonatal transport teams with logistical and ambulance resources. Developing a 'can-do' attitude and a flexible approach that focusses on added value and making a difference with what you have will ensure efficient use of limited and immature resources. During this early phase, there are advantages to be gained from building a brand, which can act as a focal point for your new team but also encourage feedback from users. Team cohesion undoubtedly benefits from the feeling of belonging and ownership which can flow from a clear brand which is enhanced by logos on paperwork, uniforms, equipment and vehicles. In the modern age, a functional website supported by social media is essential and allows stakeholders to engage. Guidelines and other cognitive aids can be hosted online, helping professionals to provide consistently high standards of clinical care. Encouraging feedback via the website is an important part of a Quality Improvement programme in the early phase and should become embedded in the 'Just Culture' of the organisation moving forwards. Stakeholder engagement and confidence is also enhanced by making safety and quality data available online. In addition to online engagement, any new service should aim to throw its doors open to visitors who will be intrigued to see the new procedures in action. Formal open days for professional and community groups work well but also one-to-one visits by key individuals who may influence opinion and/or policy will often bear fruit. Important in this early phase is a culture of listening, because this is the only way to positively change the service when it is still malleable.

\section{It is all About People}

Transport medicine is a people thing. At the heart of the process are human factors; particularly communication, teamwork and leadership. When two or more teams come together to establish a combined team, it is these human factors that will determine how successful the project is. Hopefully, there will be a bank of expertise and enthusiasm that can be drawn on and focussed by clear leadership and a drive to common goals. However, there may also be a sense of loss associated with services that have been replaced, and this could potentially hinder progress if it is not addressed and dealt with. New team members joining from a non-transport background or from other areas can potentially be positive drivers in this regard, bringing fresh perspectives and new ideas. Whatever the new dynamic, it is inevitable that the system will be stressed by the complex task ahead and the challenging scope of care that is presented by neonatal and paediatric critical care transport in a combined model. As in many aspects of medicine, it is the nursing staff that form a strong foundation, and it is on this, hopefully, stable ground that the service will be built. 
Much work has been done on the "right team" for transport. Many UK teams are using Advanced Nurse Practitioners (ANP). Data from 2003 showed in a neonatal team that an ANP model is practical and safe [20]. Practitioners had longer stabilisation times, but their patients had a better $\mathrm{pH}$ pre transfer and arrived with a better temperature.

The staffing model used at Embrace provides either Advanced Nurse Practitioners or trainee Paediatricians with a Transport Consultant overseeing the process. The trainee Paediatricians are on a rotational paediatric training scheme and the posts at Embrace have been recognised by the UK Royal College of Paediatrics and Child Health for advanced training in Paediatric Intensive Care Medicine and Neonatal Medicine.

Worldwide, there are many models of care and combinations of professionals to provide care. Registered nurses are often combined with paramedics, respiratory therapists, registered nurses or physicians. When developing specialised teams, combined or not, van Lieshout's group summarised the concept well-the leader should be same as in the NICU or PICU [21]. They compared Nurse and Paramedic teams with Nurse and Physician teams and found no significant difference in critical events.

In the UK, Advanced Nurse Practitioners generally are trained in one specialty, either neonatology or paediatric intensive care medicine. Once enrolled at Embrace, there is a well-developed training programme to ensure competencies from their "non-base" specialty. This training continues throughout their work at Embrace with honorary contracts at regional centres to allow CPD in both paediatric and neonatology as well as rostered time in anaesthesia to allow ongoing competencies in airway management.

As well as medical and nursing staff, a core administrative team are an essential part of the service. Key roles include managing referrals either online, by telephone or via telemedicine links, collecting data and publishing reports for QI.

\section{Equipment}

The concept of taking intensive care to the patient's bedside and providing mobile intensive care on route to the destination hospital is becoming a standard of care. This is reflected in the NETS (NSW) strapline 'moving intensive care for kids' which encapsulates both the practical and emotional aspects of the work. The requirement of transport equipment to provide that 'moving intensive care' is challenged by the constraints of size, weight, portability, power and safety. Combined teams with different modes of transport must strive to have, where possible, the same equipment on all the stretchers and incubators to ease the training burden and ensure high levels of expertise. Equipment that can be used across all patient ranges ensures sustained confidence and competence and reduces risk. The nirvana of stretchers and incubators that can be used across all forms of ground and air transport is difficult and expensive to reach but has many benefits. Even the most well developed air transport systems still require secondary transfers by road and the safety cost for poorly designed equipment that does not meet these challenges can be significant.

Having specialised transport equipment allowing intensive care to be taken to the patient provides the ability for effective stabilisation prior to 
moving. Recent data strengthened the argument for stabilisation compared to the previous "scoop and run" model showing that time spent undertaken intensive care interventions at the referring hospital does not worsen patient outcome [22].

Stroud et al. described how the use of high spec monitoring can improve outcome of transported patients. They focussed on improved blood pressure monitoring, which led to more intervention in terms of fluid boluses leading to less organ dysfunction and a shorter length of stay on the intensive care unit [23].

When considering financial input into a new service, specialised equipment must come high up on the list.

\section{Focussing on Safety and Quality}

Establishing a 'just culture' from the beginning will pay dividends in providing a service focussed on quality and safety. Working in the complex environment of neonatal and paediatric transport requires team members to work in a discretionary space in a flexible way conducive to good patient care but with clear boundaries set by the organisation to ensure the safety of all involved. This inevitably means there is a significant amount of process, including standard operating procedures, checklists and reporting. Identifying the sweet spot, where time efficiency, quality and safety are balanced, is challenging but worth striving for. It is inevitable that the wider a service's scope of care, the greater the pressure on the time efficiency side of this equation, potentially resulting in longer stabilisation times. This can become a patient care issue when time is a critical factor in outcome. Developing teams that can flex up to deal with these situations is imperative.

The US Institute of Medicine has outlined the six elements of a quality health care system [24]. Specifically, a quality system must deliver care that is safe, timely, effective, equitable, efficient and patient-centred.

Assessing quality begins with simple case reviews in a reflective atmosphere. At Embrace, all cases from the previous $24 \mathrm{~h}$ are reviewed by a senior clinician in a formal utilisation review process. Defined triggers for collaborative group case review are used and there is tracking of any trends in metrics such as unplanned extubation or delayed time critical transfer. Bigham and Schwartz have argued that in order to measure quality adequately, an evidence-based or consensus-driven col-

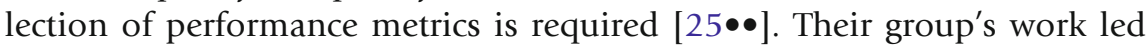
to a collaboration with the American Academy of Pediatrics' Section on Transport Medicine and the Air Medical Physicians Association to define transport metrics in the Ground and Air Medical qUality Transport (GAMUT) database which Embrace submits to [14].

Consideration should also be given to the benefits of formal accreditation. There are several international transport accreditation organisations and it is important to choose carefully to get the best fit for your service. The timing of inviting such a thorough and detailed examination of the service should also be analysed. At Embrace, we decided to apply to the Commission on Accreditation of Medical Transport Systems (CAMTS) because of its focus on developing a partnership to assist with 


\section{Consolidation}

the self-improvement of the transport team [26]. CAMTS standards cover ground and air transport and importantly the interaction between clinical teams, communication specialists, drivers, pilots and supporting staff. We took the option of requesting a consultation visit initially which allowed us to identify gaps against the standards that needed addressing before applying successfully for full accreditation in 2014 .

\section{Conclusion}

With such a major change in service provision there will be phases in the development which need to be anticipated and understood. It is inevitable that the initial years of enthusiasm and constant innovation will wane and a period of consolidation will be entered. This is a good thing in many ways, as it provides time to breathe and reflect, but not become complacent. Exploring ways to constructively challenge the system ensures the team stays updated and motivated, as long as this is balanced against the dangers of constant change.

Establishing a combined neonatal and paediatric transport system from scratch is achievable if a detailed analysis of local needs show that it is desirable and feasible. Detailed planning with adequate time and financial support is required, with involvement from all stakeholders. Systems that allow continuous review of activity and quality metrics are critical to ensure services are responsive to changing needs. Above all, leaders must remember that whatever the scope of care of a transport service, it is the patients that are the focus and the team that will provide the path to success.

\section{Compliance with Ethical Standards}

\section{Conflict of Interest}

Catherine Harrison declares no conflicts of interest.

Stephen Hancock reports and Board Member, Air Medical Physicians Association (AMPA) Ûoard Member (representing AMPA), Commission on Accreditation of Medical Transport Systems EU (www.camtseu.org).

\section{Human and Animal Rights and Informed Consent}

This article does not contain any studies with human or animal subjects performed by any of the authors.

Open Access This article is distributed under the terms of the Creative Commons Attribution 4.0 International License (http://creativecommons.org/licenses/by/4.0/), which permits unrestricted use, distribution, and reproduction in any medium, provided you give appropriate credit to the original author(s) and the source, provide a link to the Creative Commons license, and indicate if changes were made. 


\section{References}

Papers of particular interest, published recently, have been

highlighted as:

- Of importance,

• Of major importance

1. Agency for Healthcare Research and Quality; Healthcare Cost and Utilization Project (HCUP). 2012. Available at: hcup-us.ahrq.gov/.

2. Edge WE, Ranter RK, Weigle CG, Walsh RF. Reduction of morbidity in interhospital transport by specialized pediatric staff. Crit Care Med. 1994;22(7):1186-91. https://doi.org/10.1097/00003246-199407000-00023.

3. Barry PW, Ralston C. Adverse events occurring during interhospital transfer of the critically ill. Arch Dis Child. 1994;71(1):8-11. https://doi.org/10.1136/adc.71.1.8.

4. Ramnarayan P, Thiru K, Parslow RC, Harrison DA, Draper ES, Rowan KM. Effect of specialist retrieval teams on outcomes in children admitted to paediatric intensive care units in England and Wales: a retrospective cohort study. Lancet. 2010;376(9742):698-704. https://doi.org/10. 1016/S0140-6736(10)61113-0.

5. Orr RA, Felmet KA, Han Y, McCloskey KA, Dragotta MA, Bills DM, et al. Pediatric specialized transport teams are associated with improved outcome. Pediatrics. 2009;124(1):40-8. https://doi.org/10.1542/peds. 2008-0515.

6. McPherson ML, Graf JM. Speed isn't everything in pediatric medical transport. Pediatrics. 2009;124(1):3813. https://doi.org/10.1542/peds.2008-3596.

7. Vos GD, Nissen AC, Nieman FH, Meurs MM, van Waardenburg DA, Ramsay G, et al. Comparison of interhospital pediatric intensive care transport accompanied by a referring specialist or a specialist retrieval team. Intensive Care Med. 2004;30(2):302-8. https:// doi.org/10.1007/s00134-003-2066-7.

8. $\quad$ Patel SC, Murphy S; Penfil S, Romeo D, Hertzog JH. Impact of interfacility transport method and specialty teams on outcomes of pediatric trauma patients.

Pediatr Emerg Care. 2017.

Review of pediatric trauma transfers showing that air transport by generalists was faster but quality indicators suggested poorer outcomes.

9. Chamberlain G, Pearce JM. The flying squad. Br J Obstet Gynaecol. 1991;98(11):1067-9. https://doi. org/10.1111/j.1471-0528.1991.tb15355.x.

10. Britto J, Nadel S, Maconochie L, Levin M. Morbidity and severity of illness during interhospital transfer: impact of a specialised paediatric retrieval team. BMJ. 1995;311(7009):836-9. https://doi.org/10.1136/bmj. 311.7009 .836

11. Rashid A, Bhuta T, Berry A. A regionalised transport service, the way ahead? Arch Dis Child. 1999;80(5):488-92. https://doi.org/10.1136/adc.80. 5.488 .
12. Paediatric Intensive Care Audit Network (PICANet): picanet.org.uk.

13. UK Neonatal Transport Group (NTG): ukntg.net/ annual-reports/.

14. GAMUT: gamutqi.org/.

15. Embrace Yorkshire \& Humber Infant \& Children's Transport Service: sheffieldchildrens.nhs.uk/embrace/ quality-improvement/.

16. Office for National Statistics: ons.gov.uk.

17. Health Education England: hee.nhs.uk/.

18. Toolkit for high quality neonatal services, Department of Health, October 2009.

19. Quality Standards for the Care of Critically Ill Children 5th Ed 2015: picsociety.uk/wp-content/uploads/2016/ 05/PICS_standards_2015.pdf

20. Leslie A, Stephenson T. Neonatal transfers by advanced neonatal nurse practitioners and paediatric registrars. Arch Dis Child Fetal Neonatal Ed. 2003;88(6):F50912. https://doi.org/10.1136/fn.88.6.F509.

21. van Lieshout EJ, Binnekade J, Reussien E, Dongelmans $D$, Juffermans NP, de Haan RJ, et al. Nurses versus physician-led interhospital critical care transport: a randomized non-inferiority trial. Int Care Med. 2016;42(7):1146-54. https://doi.org/10.1007/ s00134-016-4355-y.

22. Borrows EL, Lutman DH, Montgomery MA, Petros AJ, Ramnarayan P. Effect of patient- and team-related factors on stabilization time during pediatric intensive care transport. Pediatr Crit Care Med. 2010;11(4):451-6.

23. Stroud MH, Prodhan P, Moss M, Fiser R, Schexnayder $S$, Anand K. Enhanced monitoring improves pediatric transport outcomes: a randomized controlled trial. Pediatrics. 2011;127(1):42-8. https://doi.org/10. 1542/peds.2010-1336.

24. Institute of Medicine. Committee on quality of health care in America; crossing the quality chasm: a new health system for the 21st century. Washington, DC: National Academy Press; 2001.

25.• Bigham MT, Schwartz HP, for the Ohio Neonatal/ Pediatric Transport Quality Collaborative. Quality metrics in neonatal and pediatric critical care transport: a consensus statement. Pediatr Crit Care Med. 2013;14(5):518-24.

Demonstrates that quality metrics can be achieved through consensus building and provides the foundation for benchmarking among neonatal and pediatric transport programs.

26. Commission on Accreditation of Medical Transport Systems: camts.org/. 